\title{
Traducir Marruecos a través del francés: el caso de los textos registrales
}

\author{
Tanagua BARCELÓ MARTÍNEZ \\ Universidad de Málaga \\ tbmartinez@uma.es \\ https://orcid.org/0000-0001-8688-5580
}

\section{Resumen}

La coexistencia de diferentes lenguas en un mismo territorio es un acontecimiento apasionante fruto de la historia y de las relaciones habidas entre las civilizaciones. En la práctica, esta circunstancia tiene consecuencias sociolingüísticas y traductológicas y, por ello, es necesario ahondar en su estudio con el objeto de dar respuesta a las distintas problemáticas que se puedan derivar de este fenómeno. En este caso, trataremos de analizar el contexto, las condiciones y las consecuencias de la traducción al español de documentos registrales procedentes de Marruecos redactados en francés. Para ello, expondremos cuál es la situación lingüística en Marruecos, cuál es el uso y por qué de la lengua francesa y qué consecuencias tiene en la traducción de los mencionados documentos.

Palabras clave: relación hispano-marroquí, francés lengua pivote, política lingüística, textos registrales.

\section{Résumé}

La coexistence de différentes langues sur un même territoire est un évènement passionnant résultant de l'histoire et des relations qui existent entre les civilisations tout au long de l'histoire. Dans la pratique, cette circonstance a des conséquences sociolinguistiques et traductologiques dont l'étude est nécessaire afin de donner une réponse aux problèmes qui pourraient en découler. En l'occurrence, nous tenterons d'analyser le contexte, les conditions et les conséquences de la traduction en espagnol des documents des différents registres procédant du Maroc et rédigés en français. Pour ce faire, nous analyserons la situation linguistique au Maroc, l'emploi de la langue française dans ce pays et les conséquences de ceci pour ce qui est de la traduction des documents mentionnés.

Mots clé : relation hispano-marocaine, français langue pivot, politique linguistique, textes du registre.

\begin{abstract}
The coexistence of different languages in a particular territory is a fascinating event that stems from history and the relations of any kind, built up among civilizations. In practice,
\end{abstract}

*Artículo recibido el 11/07/2020, aceptado el 17/11/2020. 
this circumstance has both sociolinguistic and translation consequences and, thus, it is necessary to delve into their study in order to respond to problems emerging from this phenomenon. In this case, we shall try to analyse the context, the conditions and the consequences of the translation into Spanish of Moroccan registry documents written in French. To this end, we shall present the linguistic status of Morocco, the reasons behind its use of the French language and its effects on the translation of the aforementioned documents.

Keywords: Spanish-Moroccan relations, French as a bridging language, language policy, registry texts.

\section{Introducción}

Los vínculos existentes entre Marruecos y España datan de largo tiempo. La proximidad geográfica, entre otros factores, ha propiciado que ambos países mantengan una estrecha relación a todos los niveles que, hoy en día, persiste. En la actualidad, podemos afirmar que las relaciones hispano-marroquíes gozan, en general, de buena salud. Ambos países son uno de los principales socios comerciales el uno del otro (excluyendo, en el caso de España, los países de la Unión Europea), lo que ha multiplicado exponencialmente tanto el flujo de transacciones comerciales como el movimiento migratorio. En ese contexto, el papel de la traducción resulta crucial, ya que posibilita un mayor y mejor entendimiento y permite la realización de muchos trámites, como bien expone Faddi (2015: 62) al manifestar que

la figura del migrante es un símbolo de nuestros tiempos y su figura es objeto de muchas reflexiones en distintas disciplinas, entre ellas la traductología. Las convulsiones que experimenta nuestra era necesitan la traducción hoy más que nunca, lo que hace del traductor, como persona subjetiva, una pieza clave.

En ese sentido, y tal y como afirma Ruiz Cortés (2019: 223), «uno de los primeros contactos que un migrante tiene con la sociedad de acogida es a través de su Administración, dada la necesidad de adquirir la documentación administrativa necesaria para acreditar su residencia». Entre los documentos más frecuentemente demandados y, por lo tanto, traducidos, se encuentran los denominados textos registrales, que, de algún modo, hacen las veces de "carné de identidad» de las personas, ya que contienen los datos básicos relativos a su estado civil, sus antecedentes penales, sus bienes, sus sociedades, etc.

En las relaciones entre Marruecos y España se produce un fenómeno lingüístico de gran interés cuyo estudio puede ser abordado desde numerosas ópticas. Nos referimos al uso de la lengua francesa que Marruecos hace en la redacción y expedición de documentos jurídico-administrativos (registrales, en este caso) cuya presentación (y, por lo tanto, cuya traducción) es un requisito indispensable para la entrada y la regularización de ciudadanos marroquíes en nuestro país. Esta realidad sociolingüística solo 
se justifica si echamos la vista atrás y tenemos en cuenta los tres aspectos fundamentales que la sustentan: la historia, la normativa y la situación actual. Ello impone un acercamiento transversal al tema en cuestión, en la línea de estudios recientes que vinculan la traducción con la migración, algunos de los cuales mencionaremos más adelante. El hecho de que, para sus relaciones con España, Marruecos anteponga en muchas ocasiones el uso del francés no solo responde a razones lingüísticas, sino que han de tenerse en cuenta factores de índole política, cultural, económica y religiosa, entre otros, que justifican la situación, la variedad y la política lingüística de cada territorio.

\section{Objetivos}

Partiendo del contexto anteriormente expuesto y conscientes de que puede ser abordado desde diferentes ópticas y ámbitos del saber, nos proponemos en esta ocasión los siguientes objetivos, que giran en torno a los tres aspectos evocados: historia, normativa y situación actual.

En primer lugar, se realizará un breve recorrido por los acontecimientos históricos que justifican la actual situación sociolingüística de Marruecos, con especial hincapié en la lengua francesa. En segundo lugar, se intentará justificar dicha situación a partir de las principales fuentes normativas que actualmente regulan la política lingüística en Marruecos. En tercer y último lugar, nos trasladaremos al ámbito de la traductología para comprobar qué repercusión puede tener todo lo anterior en la traducción hacia el español de textos registrales marroquíes y la importancia de la presencia del francés en dicho proceso.

En este trabajo se combinan, pues, diferentes acercamientos. Por un lado, se trata de un estudio de corte descriptivo fruto del estudio, análisis y resumen de la información necesaria que contribuye a conformar el contexto que justifica la existencia de la realidad lingüístico-traductológica a la que aquí pretendemos aludir. Por otro lado, el uso de un método con orientación cuantitativa a partir del análisis y la interpretación de datos estadísticos resultantes de encuestas creadas ad hoc nos permite sustentar nuestro trabajo y extraer una serie de conclusiones. Todo ello nos lleva a una reflexión acerca del fenómeno sociolingüístico y traductológico abordado y sus consecuencias $^{1}$ a partir de la investigación y de la propia experiencia en el ámbito en cuestión.

\section{Antecedentes}

Como prueba del interés que suscita el tema que aquí pretendemos abordar, debemos mencionar a algunos autores que se han preocupado por adentrarse en el estudio y el análisis de diferentes aspectos relacionados con la traducción de documentos jurídico-administrativos procedentes de Marruecos.

\footnotetext{
${ }^{1}$ En esta ocasión nos limitaremos a la situación en Andalucía por ser nuestro punto de referencia desde la experiencia del traductor jurado y por la más que estrecha relación entre dicha comunidad autónoma y el país vecino.
} 
Así, los autores que a continuación se mencionan han llevado a cabo investigaciones que han dado lugar a trabajos relacionados con la traducción de este tipo de documentos, en general, y de los textos registrales, en particular, procedentes de $\mathrm{Ma}$ rruecos o de países africanos francófonos. Algunos de ellos, además, se refieren al uso del francés como lengua intermediaria en la traducción de documentos hacia el español. La mayoría de estos estudios se enmarcan o aluden al ámbito de la traducción jurada, por ser aquel en el que se suele llevar a cabo la traducción de los documentos que aquí nos ocupan. Entre dichos autores, destacamos a Aguessim El Ghazouani (2013), Barceló Martínez y Delgado Pugés (2014), Bouteffah Aghbalou (2017), Casas Cabido (2000), Feria García (1999), Kadiri Hassani (2015), Karim (2010) y Pérez de Gracia Sánchez (2018), entre otros.

En cuanto a estudios de corte más general que dejan de lado el aspecto traductológico y que abordan la situación lingüística de Marruecos o de los demás países del Magreb y sus causas desde un punto de vista político, cultural, religioso y/o cultural, debemos decir que son muy numerosos. Mencionamos trabajos como los de Abbou (1984), Achouche (1981), Afeli (1990), Akouaou (1984, 1997), Benítez Fernández (2006, 2012) o Moustaoui (2007), entre otros muchos.

Nuestra investigación podría enmarcarse en el grupo de trabajos que pretenden combinar aspectos sociales (relacionados, sobre todo, con la inmigración), por un lado, y lingüístico-traductológicos, por otro, es decir, aquellos que ponen de relieve la función social de la traducción. En ese sentido, destacan, entre otros, los trabajos de ElMadkouri Maataoui (2010), El-Madkouri Maataoui y Soto Aranda (2012) o Ruiz Cortés (2019).

\section{La política lingüística en Marruecos}

Antes de referirnos concretamente al caso de Marruecos, conviene definir qué entendemos por política lingüística. Según Aguilera Martín (2003: 91), «[1]a política lingüistica estaría constituida por el conjunto de ideas, leyes, regulaciones y prácticas que se dirigen a producir cambios en los comportamientos lingüísticos de una sociedad o de un grupo social». Este concepto se diferencia del de planificación lingüística, con el que a menudo se confunde. Este último, y siempre según Aguilera Martín (2003: 91), «haría referencia al conjunto de decisiones adoptadas por una autoridad, en principio gubernamental, para conseguir estos mismos resultados ${ }^{2} »$.

\footnotetext{
2 Por su parte, Moustaoui Srhir (2007: 72) define la política lingüística como «el conjunto de declaraciones de intenciones, decisiones e ideas conscientes realizadas en el ámbito de las relaciones de la lengua o lenguas con la vida social y cuyos objetivos serían producir cambios en la situación y los comportamientos lingüísticos de una sociedad o de un grupo social determinado». En cuanto a la planificación lingüística, el autor señala que «es uno de los aspectos de la política lingüística que consiste en la puesta en práctica de dichas decisiones a través de distintos procesos de regulación y, por lo tanto, tiene una función intervencionista y práctica».
} 
Tanto la política como la planificación lingüísticas no son acciones arbitrarias, sino que están motivadas por factores históricos, políticos, religiosos, culturales y económicos, y son, pues, fruto de los acontecimientos y de la situación de cada Estado. Ello provoca, en ocasiones, conflictos relacionados con el uso de determinadas lenguas en determinados territorios, por lo que resulta imperativo repasar los hechos que explican su situación sociolingüística.

En cuanto a la situación lingüística del país vecino, esta presenta una cierta complejidad, ya que se trata de un país en el que (co)existen y se hablan varias lenguas y cada una de ellas posee un estatus diferente debido, principalmente, a que han ido apareciendo en el territorio en diferentes etapas o momentos de su historia. Como señala Moustaoui Srhir (2007: 71), los trabajos sobre política lingüística y las medidas llevadas a cabo en Marruecos surgieron durante el proceso de descolonización del país, por lo que gran parte de los estudios en torno a estos conceptos están estrechamente ligados con el proceso de independencia y la construcción del nuevo Estado. La política lingüística llevada a cabo en Marruecos desde su independencia ha ido evolucionando paulatinamente $y$, aunque ha estado siempre enfocada a la arabización total del país, esta evolución se podría dividir en dos etapas, que se corresponden también con los reinados de Hassan II y Mohammed VI.

La primera de ellas se corresponde con el modelo de política lingüística implantado en Marruecos tras su independencia. Esta etapa se caracterizó principalmente por el deseo de este nuevo Estado de conservar el árabe clásico en un intento por mantener los lazos de unión entre dicha lengua y la religión islámica, y entre la religión y la monarquía. Así, en referencia a la monarquía islámica, y señalando que esta representa también un símbolo de unidad frente al contexto de diversidad y pluralidad lingüística, Moustaoui Srhir (2007: 193) indica de este modo cuál fue la política lingüística establecida por el Estado marroquí:

A partir de estos factores, Marruecos estableció, primero, su Constitución y su Madawana [Código Civil] donde dio primacía al derecho islámico $[\ldots]$ y, segundo, la elección del árabe clásico como lengua oficial, cuando el árabe marroquí es la variedad materna de la mayoría.

Este primer modelo de política lingüística trajo consigo el establecimiento de escuelas coránicas en el país, la introducción y estudio, como asignatura obligatoria, de las ciencias islámicas, en el ámbito de la educación y, sobre todo, un proceso de arabización en la administración pública y la enseñanza, lo que propició el uso del árabe clásico en los ámbitos político e institucional Sin embargo, cabe destacar, como señala también Moustaoui Srhir (2007: 193), que, pese a esta política lingüística, en aquella etapa se siguió utilizando el francés: «[e]l mantenimiento del francés y el empeño de las instituciones políticas por conservarlo demuestran que esta lengua era y es más que una 
herencia de la colonización, es un patrimonio cultural que se ha impuesto entre la población».

Podríamos resumir esta primera fase haciendo hincapié en la ideología nacionalista árabe-marroquí como modelo de política lingüística y destacando los tres puntos que señala Moustaoui Srhir (2007: 194):

- Se defiende el monolingüismo absoluto, y ello se plasma en la elección del árabe clásico como lengua oficial del Estado. A pesar de ello, el peso del francés sigue siendo notorio y dicha lengua no deja de utilizarse.

- Se incide en una historia única dando prioridad a un grupo mayoritario y olvidando otros (como sería el caso de los bereberes, por ejemplo, y su lengua amazigh).

- Se insiste en la exaltación del pasado histórico de la lengua dominante: el árabe clásico.

La segunda etapa se refiere al nuevo modelo de política lingüística, que comenzó tras la subida al trono de Mohammed VI en 1999 y que todavía hoy perdura. Este acontecimiento trajo consigo numerosos cambios para el país y su relación con el resto del mundo. Quizá uno de los más destacados fue el cambio de discurso sobre la política lingüística y la valoración de las lenguas existentes en el territorio marroquí. A través de dicho discurso comenzó a aceptarse la existencia del multilingüismo en Marruecos. Así, esta nueva política dio lugar al reconocimiento del amazigh como lengua oficial del Estado, hecho apoyado por la nueva legislación y las instituciones oficiales encargadas de poner en marcha este nuevo modelo.

\subsection{Normativa en materia de política lingüística en Marruecos}

Tal y como hemos avanzado en la exposición de nuestros objetivos, conocer cuáles son las fuentes que regulan la política lingüística de Marruecos nos permitirá entender la presencia y el peso del francés en la expedición de determinados documentos y sus posibles consecuencias de cara a la traducción. Así, en el estado de cosas descrito, son varios los textos normativos sobre los que se sustenta la política lingüística del Estado marroquí, entre los que destacamos los que siguen:

- La Constitución marroquí de $2011^{3}$ (Royaume du Maroc, 2011), por la que se reconocen dos lenguas oficiales, el árabe clásico y el amazigh, así como sus diferentes variantes dialectales.

- La Charte nationale d'éducation et de formation (Royaume du Maroc, 1999). En palabras de Moustaoui Srhir (2007: 280), «se podría considerar como una carta magna dentro del sistema educativo marroquí». En ella no solo se establece el carácter oficial del árabe, sino su obligatoriedad en la enseñanza de

\footnotetext{
${ }^{3}$ La versión de la Constitución marroquí de 1996 únicamente reconocía el árabe clásico como lengua oficial del Estado y excluía el amazigh o bereber e incluso el árabe marroquí, variedad desprotegida y olvidada de la política lingüística marroquí pese a ser la lengua materna de la mayoría de la población.
} 
todos los marroquíes, aunque hace énfasis en la variedad de árabe marroquí. Además, este documento alude también a la creación de la Academia de la Lengua Árabe y reconoce el amazigh como lengua de enseñanza, aunque sin precisar su estatus sociolingüístico. Este documento también hace referencia a las lenguas extranjeras y a la importancia de dominar su uso.

- El Dahir no 1-01-299 portant création de l'Institut royal de la culture amazigue (IRCAM) (Royaume du Maroc, 2001b), du 17 octobre 2001 (29 rajab 1422). Este decreto, además de reconocer la diversidad lingüística de Marruecos, es uno de los textos más importantes sobre política lingüística respecto al amazigh, ya que gracias a él se crea el Institut royal de la culture amazigue en Marruecos (IRCAM) y se establecen medidas de planificación lingüística.

- La Loi no 01-00 portant l'organisation de l'enseignement supérieur du ler juin 2000 (Royaume du Maroc, 2000). Desde la independencia de Marruecos, su política lingüística en el ámbito universitario se ha basado en un bilingüismo que combina tanto el árabe clásico como el francés. Generalmente, la tendencia era a privilegiar más el árabe clásico en la enseñanza de las facultades de letras y el francés - y, en ocasiones, el inglés - en las ciencias (Moustaoui Srhir, 2007: 283). Sin embargo, esta ley, pese a reconocer la importancia del francés y del árabe clásico en la enseñanza universitaria, también reconoce el amazigh y se hace eco de la importancia de realizar estudios de corte lingüístico-cultural sobre esta lengua.

En resumen, observamos que Marruecos únicamente reconoce en su carta magna dos lenguas oficiales, así como diversas variantes dialectales, pero otorga una importancia destacable al empleo de la lengua francesa, sobre todo en el ámbito educativo y, como veremos más adelante, en el de la administración pública.

\section{Situación lingüística actual en Marruecos}

La situación lingüística de cada territorio está, pues, fuertemente influida por los conflictos, las guerras, las colonizaciones o los procesos de independencia y anexión, entre otros acontecimientos, que han propiciado la coexistencia de dos o más lenguas y han dado lugar a panoramas lingüísticos variados en la actualidad. Esto acarrea consecuencias de diversa índole que tienen que ver con factores identitarios y culturales vehiculados a través de las lenguas. Así, podemos afirmar que, a pesar de que son muchos los países que solo contemplan en su legislación una lengua oficial, no es menos cierta la (co)existencia de otras lenguas y dialectos que conviven, con diferentes estatus, en una determinada zona ${ }^{4}$. Es, como hemos visto, el caso de Marruecos, cuya historia

\footnotetext{
${ }^{4}$ Algunos ejemplos son países como Canadá (que cuenta con el francés y el inglés como lenguas oficiales y donde, además, coexisten dos sistemas jurídicos: el derecho anglosajón o common law, que constituye el derecho federal, por un lado, y el derecho continental, aplicado en la provincia de Quebec, por otro), Suiza (con cuatro lenguas oficiales: alemán, francés, italiano y romanche) o India (cuyas lenguas oficiales
} 
y tradición han llevado a este país a convivir con diferentes lenguas y dialectos ( $c f$. Bouziane, 2018; Quitout, 2001) que han adquirido más o menos importancia política y social en función de la época y que han dado lugar a una riqueza lingüística digna de mención y que aún perdura.

La Constitución marroquí (Royaume du Maroc, 2011), en su artículo quinto, reconoce dos lenguas oficiales: el árabe y el amazigh o bereber. Ambas cuentan con diferentes formas dialectales. En ese mismo artículo ${ }^{5}$ y con una clara vocación de apertura al mundo occidental, también se establece que:

L'État œuvre à la préservation du Hassani, en tant que partie intégrante de l'identité culturelle marocaine unie, ainsi qu'à la protection des parlers et des expressions culturelles pratiqués au Maroc. De même, il veille à la cohérence de la politique linguistique et culturelle nationale et à l'apprentissage et la maîtrise des langues étrangères les plus utilisées dans le monde, en tant qu'outils de communication, d'intégration et d'interaction avec la société du savoir, et d'ouverture sur les différentes cultures et sur les civilisations contemporaines.

El árabe estándar o clásico es, junto con el francés, la lengua de la enseñanza, de los medios de comunicación y de la administración. Es también la lengua de lo sagrado, del islam, de la oración y de los rituales religiosos. No es, sin embargo, la lengua hablada por la mayoría de los marroquíes, que es el árabe marroquí. Desde hace algún tiempo, otras lenguas extranjeras, como el español y el inglés, han cobrado una cierta relevancia en Marruecos, con un uso creciente debido a las relaciones y transacciones entre los diferentes países cuyas lenguas son algunas de las citadas. Según Moustaoui Srhir (2007: 159), existe incluso un "conflicto de intereses socioeconómicos entre la Francofonía, la Hispanofonía y la Anglofonía».

\subsection{Presencia e importancia del francés en Marruecos}

Con respecto a la Francofonía, es bien sabido que la presencia y el uso del francés más allá de Francia no es un hecho aislado. Esta lengua está presente, en mayor o menor grado y por diferentes razones, en muchos países del mundo, lo que la convierte en una de las más influyentes que existen. La justificación hunde sus raíces en la historia

son el hindi y el inglés, pero donde 22 lenguas son reconocidas como cooficiales por ser las más habladas según la provincia de que se trate).

${ }^{5}$ La cita se corresponde con el quinto párrafo del artículo 5 de la versión francesa de la Constitución de Marruecos, cuya traducción al español proponemos como sigue: «El Estado vela por la preservación del hassanía, en tanto que parte integrante de la identidad cultural marroquí unida, así como por la protección de los dialectos y de las expresiones culturales que se practican en Marruecos. De igual modo, vela por la coherencia de la política lingüística y cultural nacional y por el aprendizaje y el dominio de las lenguas extranjeras más utilizadas en el mundo, en tanto que útiles de comunicación, de integración y de interacción con la sociedad del conocimiento y de apertura sobre las diferentes culturas y civilizaciones contemporáneas». 
y las relaciones político-culturales habidas, que han dejado diferentes huellas lingüísticas según el país. El francés es la lengua oficial o cooficial de numerosos Estados y una de las lenguas oficiales de organismos e instituciones como la Unión Europea o la Organización de Naciones Unidas. Según un estudio del año 2018 realizado por el Observatoire de la langue française (Organisation internationale de la Francophonie, 2018), se estima que actualmente existen unos 300 millones de francófonos en el mundo, y se prevé que dicha cifra no deje de aumentar.

Con respecto al reparto mundial de francófonos, el mismo estudio muestra la presencia especialmente notoria del francés en África ${ }^{6}$ como consecuencia de los procesos de colonización de numerosos países por parte de Francia entre los siglos XVI y XIX. No obstante, conviene matizar que la lengua francesa tiene diferentes estatus según el país o estado de que se trate. Así, es lengua oficial en países como Francia, Mónaco, Mali, o Costa de Marfil; lengua cooficial en Bélgica, Suiza, Luxemburgo, Camerún, Canadá o Madagascar; lengua administrativa en Marruecos, Argelia, Mauritania, Líbano, etc.

En el caso concreto de Marruecos, según el estudio antes aludido, el número de hablantes de francés es de 12729000 (de un total de 36,5 millones de habitantes, lo que supone el 34,8 \% de la población marroquí). A pesar de no ser una lengua oficialmente reconocida, el francés tiene hoy en día un importante prestigio en Marruecos y no se puede obviar la destacada presencia en ámbitos tan importantes de la vida pública como la enseñanza, la administración o la justicia. Se emplea también frecuentemente en sectores como el comercio, la diplomacia o los asuntos gubernamentales, además de como lingua franca o vehicular. En ese sentido, no sería aventurado afirmar que el francés es la segunda lengua empleada en Marruecos.

Pero ¿cómo, cuándo y por qué llega el francés a Marruecos? Tal y como afirma Quitout (2001: 62),

[d]epuis la signature du traité de Fès le 30 mars 1912 jusqu'à la proclamation de l'indépendance le 2 mars 1956, la langue française était la langue officielle du régime du Protectorat et de ses institutions. Après cette date, le français a gardé un rôle privilégié en ce sens qu'il est considéré comme la première langue étrangère du pays. Malgré la vague d'arabisation que l'on connaît, le français a continué et continue encore de se maintenir dans des proportions considérables.

Esta afirmación deja claro el rastro del francés en Marruecos, que, lejos de desaparecer tras la descolonización, se ha convertido (aunque no de forma oficial) en la

\footnotetext{
${ }^{6}$ En el continente africano, el francés permite viajar de Marruecos a Madagascar, una superficie de unos 13 millones de $\mathrm{km}^{2}$. La llamada África francófona es, por tanto, un vasto territorio con lo que podríamos denominar una lingua franca común, lo que hace que el francés represente una gran ventaja para la comprensión mutua y para el comercio en esta zona.
} 
lengua de la cultura, de la diplomacia y de muchas relaciones administrativas con el resto del mundo o, cuando menos, con Europa.

Los contactos entre Francia y Marruecos se remontan, sin embargo, al Imperio romano, aunque la presencia y la influencia de la lengua francesa en dicho país por encima de las demás lenguas europeas es un fenómeno relativamente reciente cuya importancia data de principios del siglo XX7. Así, en 1906 tiene lugar la Conferencia Internacional de Algeciras, que culminó con el establecimiento de un protectorado por parte de Francia (en la zona sur) y España (en la zona norte). En 1912, la mayor parte de Marruecos se convirtió oficialmente en un protectorado francés, lo que propició que el francés pasase a ser la lengua de la administración, del gobierno, de la educación y de los medios de comunicación, relegando así el árabe clásico para las actividades tradicionales y de carácter religioso. En 1956, tras la proclamación de la independencia de Marruecos, el francés pierde su carácter oficial y comienza así un proceso de arabización. A pesar de ello, el francés no ha desaparecido ni ha dejado de utilizarse. Muy al contrario, el estado marroquí lo sigue empleando como lengua diplomática internacional y en la enseñanza pública superior. Goza, en cierto modo, de un prestigio social que no tiene el árabe, actúa como puente hacia Occidente y facilita en gran medida las relaciones internacionales. Esta idea se recoge en un informe elaborado en $2014^{8}$, en el que se afirma que

[...] el árabe clásico se habla únicamente en ciertos contextos formales muy restringidos, mientras que el francés, tanto oral como escrito, se emplea en casi todos los folletos y documentos escritos, en el sector privado y en las escuelas superiores y facultades de ciencias. En todos estos ámbitos, el francés es el idioma más utilizado y se considera la variedad alta y dominante con respecto al árabe clásico y, por supuesto, al resto de las variedades. A esta situación habría que añadir otra de carácter ideológico, y es que, en el imaginario marroquí, el francés está relacionado con el acceso a las ciencias y a la modernidad, mientras que

\footnotetext{
${ }^{7}$ Algunos autores, como Manzano (1996: 16), afirman, sin embargo, que la presencia del francés es muy anterior: «Tout d'abord, certaines études tendent à montrer que le français était utilisé avant l'acte politique de la colonisation. Dans les élites et la bourgeoisie commerçante certainement, plus généralement au sein de groupes et chez des individus en contact avec la France et l'Europe. D'autre part, réduire la francisation à la colonisation fait perdre de vue que le contact avec la Romania, avec des hauts et des bas, n'a jamais été interrompu. De ce point de vue, le Maghreb, de longue date et aujourd'hui encore, est situé sur une ligne de faille entre la famille romane et la famille chamito-sémitique».

${ }^{8}$ Fernández Vítores y Benlabbah, con la colaboración de los ministerios de Asuntos Exteriores y de Cooperación de España y Marruecos, los ministerios de Educación de España y Marruecos, la Agencia Española de Cooperación Internacional para el Desarrollo y el Instituto Cervantes, elaboraron dicho informe, en el que se alude a los diferentes conflictos lingüísticos acaecidos durante las últimas décadas en Marruecos fruto de la situación política.
} 
el árabe clásico suele asociarse al pasado y a los valores religiosos

(Fernández Vítores y Benlabbah, 2014: 22).

Esta importancia del francés en determinados sectores no está exenta, sin embargo, de polémica. Así, existen particulares y asociaciones que juzgan ilegítimo el uso de esta lengua en la Administración Pública y han llevado el asunto ante los tribunales, que han fallado en su favor. El Collectif national de la langue arabe llegó incluso a afirmar que dicho fallo representa una «victoire face au lobby francophone qui ne cesse de trouver de nouvelles entraves pour détourner, voire violer, toutes les lois liées à l'usage et à la préservation de la langue arabe» (Le Site Info, 2018). Los defensores de esta idea denuncian que esta práctica va en contra de la soberanía del pueblo, democráticamente plasmada en la Constitución marroquí ${ }^{\prime}$, que establece el árabe y el amazigh como únicas lenguas oficiales del país. El uso del francés sería, pues, anticonstitucional desde esta perspectiva. Podríamos concluir diciendo que existe en Marruecos una postura más tradicional ${ }^{10}$, que aboga por la defensa de las lenguas propias del país, en consonancia con la carta magna, y otra que ve en el uso del francés un signo de modernidad y de apertura al mundo. El francés es, al mismo tiempo, lengua privilegiada y lengua rechazada y su estatus no deja de ser opaco ${ }^{11}$.

\subsection{Otras lenguas presentes en Marruecos}

Además del árabe, del francés y de los diferentes dialectos, el español y el inglés cuentan, por diferentes motivos, con cierta importancia en Marruecos. La presencia del español data del siglo XV (Quitout, 2001: 63): «La rencontre de la langue espagnole avec le territoire marocain remonte au lendemain de la chute de Grenade en 1492 avec l'arrivée des Mauresques et des Juifs chassés d'Espagne», aunque se vio reforzada como consecuencia de la colonización española de algunas zonas del actual Marruecos (el denominado protectorado español en Marruecos). Tras la independencia y la unificación marroquí, el francés ocupó el lugar del español en todo el país como lengua de la administración y la educación, aunque este sigue empleándose en los intercambios económicos y comerciales.

El uso creciente del inglés obedece al número cada vez mayor de turistas no francófonos que visitan Marruecos, al dominio del inglés como lengua diplomática y científica internacional y a su presencia en los medios de comunicación; en definitiva, al estatus de lingua franca que parece ostentar a nivel internacional.

\footnotetext{
${ }^{9}$ Otros, sin embargo, ven en la Constitución marroquí un guiño oculto al francés en virtud del contenido de su artículo 5, antes mencionado.

${ }^{10}$ Esta postura va más allá y pone de relieve las consecuencias de la imposición del uso del francés en la sociedad. En ese sentido, El Couri (2013: 238) afirma que «la suprématie du français dans l'administration et la vie publique a affecté psychologiquement le citoyen, atteint de snobisme et de complexe d'infériorité, qui croit que parler français le rend plus civilisé».

11 Mencionamos trabajos a este respecto realizados por autores como Benzakour (2010 y 2012), Bourdereau (2006), De Poli (2005) y Messaouidi (2010), entre otros.
} 


\section{Los textos registrales y el mercado profesional}

Una vez establecido el marco sociolingüístico de Marruecos, abordamos la parte traductológica. La elección de los textos registrales en esta ocasión no es arbitraria y, entre otros factores, obedece a la demanda del mercado profesional, en el que la traducción de estos documentos supone un volumen de trabajo nada desdeñable para el traductor, especialmente para el traductor jurado, tal y como afirma Borja Albi (2007: 189): «La traducción de certificados de nacimiento, defunción o matrimonio ${ }^{12}$ [...] ocupa un lugar relevante dentro de la actividad profesional del traductor jurídico y jurado».

En un estudio reciente de Barceló y García (2018: 282) llevado a cabo a partir de la información extraída de una encuesta realizada a traductores jurados de Andalucía $^{13}$, se obtuvieron datos acerca del volumen de trabajo que supone la traducción de documentos registrales. Según se desprende del gráfico 1, los textos registrales son los segundos más traducidos después de los documentos académicos y con clara diferencia con respecto al resto de textos.



Gráfico 1. Documentos objeto de traducción jurada más demandados según la encuesta de Barceló y García (2018: 280)

Otro dato importante lo muestra el gráfico 2, según el cual el principal cliente es el particular (en un $60 \%$ de los casos). Este dato sería indicativo del movimiento migratorio existente entre Marruecos y España.

\footnotetext{
${ }^{12}$ A pesar de que un texto registral es cualquier texto que emane de cualquier registro, la realidad es que los textos que se traducen con mayor asiduidad son los procedentes del Registro Civil y del Registro Central de Penados (o sus equivalentes en cada país).

${ }^{13}$ El estudio se limitó a la Comunidad Autónoma andaluza por ser el espacio de desempeño de la actividad profesional en el que tenemos experiencia y por la proximidad con Marruecos, lo que propicia las relaciones y el intercambio de documentación. Por ese motivo, los datos no pueden aplicarse de forma general. A pesar de ello, la práctica profesional nos lleva a afirmar que los datos, en general, podrían ser extrapolables.
} 




Gráfico 2. Tipos de clientes más frecuentes de las traducciones juradas según la encuesta de Barceló y García (2018: 283)

La suma de las dos afirmaciones anteriores muestra que el particular en posesión de documentos relativos a su persona (hagan estos referencia a su estado civil, a sus bienes, a sus últimas voluntades, a la existencia o no de condenas en su contra, etc.) es quien, por lo general, solicita de forma directa al traductor la traducción de dicha documentación, cuya presentación suele ser obligatoria para cumplir con los trámites básicos para la entrada y estancia más o menos permanente y regular en España. En otros casos, esta gestión se hace a través de profesionales u organismos autorizados (abogados o agencias y oficinas especializadas en asuntos de extranjería).

\section{El francés en la Administración Pública marroquí}

Una vez que conocemos cuál es el estatus general del francés en Marruecos, damos paso a las consecuencias que este fenómeno tiene para la traducción, con especial atención a la Administración Pública y a los textos registrales. En lo relativo al ámbito de la administración, y aunque se considera que es un sector en el que el proceso de arabización se realizó de manera ejemplar, Benítez Fernández (2006: 114) indica que «'administration marocaine est un secteur dont on a l'habitude de dire qu'il est assez bien arabisé. La réalité est qu'on ne trouve presque pas de mesures mises en pratique à cet égard». Y añade:

La quasi-totalité des documents avec lesquels on communique avec l'administration ou vice-versa sont publiés en édition bilingue [árabe y francés] à commencer par le Bulletin Officiel du Maroc et, dans certains cas, ils sont publiés uniquement en français ; c'est le cas du Bilan des Impôts. Lorsqu'il s'agit de la langue parlée, c'est le cas des réunions administratives, la langue employée est généralement le français ou encore un arabe dialectal assez cultivé incrusté de termes de l'arabe classique (Benítez Fernández, 2006: 114). 
Es necesario referirse aquí al proceso de modernización de la administración iniciado y puesto en marcha por Marruecos hace ya algunos años, tal y como se refleja en las palabras del ministère de la Fonction publique et de la Reforme administrative marroquí (Royaume du Maroc, 2019: 9) cuando afirma que

[1]es défis de l'ère de la mondialisation, la construction inéluctable du Maghreb Arabe, les besoins de la décentralisation et de la déconcentration exprimée tant par les pouvoirs locaux que par les usagers, la consolidation des fondements d'un Etat de droit, constituent autant d'éléments qui appellent à la reconsidération du rôle et des missions de l'Etat et par conséquent à la modernisation de son mode de fonctionnement.

En ese proceso se han puesto en marcha numerosas iniciativas, entre las que destaca la actualización y la accesibilidad de los contenidos de las páginas webs de los organismos de la Administración Pública marroquí, que suelen ofrecer la información en árabe, amazigh (lenguas oficiales), francés e inglés, lo que muestra una clara vocación de apertura y de internacionalización.

\subsection{El francés en los textos registrales marroquíes}

Lo expuesto hasta ahora en cuanto a la importancia del francés en Marruecos, en general, y en los documentos de carácter administrativo, en particular, permite, pues, justificar (independientemente de la legitimidad que se le otorgue a este hecho) la presencia de dicha lengua en documentos emanantes de los principales organismos públicos marroquíes, entre ellos, los diferentes registros. Esta realidad se recoge, además, en diferentes textos normativos, como el Dahír n. ${ }^{\circ} 1-02-239$ de 25 rayab de 1423 (3 de octubre de 2002), que promulga la Ley n. $37-99$, relativa al estado civil en Marruecos, la cual establece que los documentos registrales deben estar transcritos a caracteres latinos. Aunque, ciertamente, el texto no se refiere a ninguna lengua en concreto, en la práctica, la lengua empleada es el francés. Más adelante haremos alusión a los documentos registrales que más se traducen entre Marruecos y Espańa y que suelen aparecer redactados en francés.

Con respecto al texto registral, en esta ocasión nos centramos en el que procede de algún registro público, y más concretamente, los emitidos por el Registro Civil, el Registro Central de Penados, el Registro Central de Actos de Última Voluntad, el Registro Mercantil y el Registro de la Propiedad (o sus equivalentes según el país). Estos organismos forman parte de la Administración Pública y los documentos que expiden son de demanda y uso cotidiano por parte de los ciudadanos ${ }^{14}$.

\footnotetext{
14 «El Registro Público, o las oficinas de registro, son los lugares que puede utilizar el ciudadano para presentar las solicitudes, escritos y comunicaciones que van dirigidos a las Administraciones Públicas. Asimismo, es el lugar que utiliza la Administración para registrar los documentos que remite al ciudadano, a entidades privadas o a la propia Administración» (Parainmigrantes, 2015).
} 
En ese sentido, los textos registrales son documentos jurídico-administrativos que «tienen serias repercusiones de orden social, en la medida en que es muy difícil que un ciudadano común no se enfrente en algún momento de su vida a la redacción o a la interpretación de un texto de este tipo» (De Miguel Aparicio, 2000: s.p.). Estos textos poseen unas características específicas que los dotan de identidad propia. De forma general, y tal y como Borja Albi (2007: 208) afirma:

$[\ldots]$ los documentos registrales presentan una disposición preestablecida del contenido que responde a una serie de formalidades determinadas, pues se trata de estructuras fijadas por la tradición, el uso y, en ocasiones, por la legislación. Se caracterizan por presentar fórmulas estereotipadas, así como por elementos culturales (instituciones, organismos, cargos, topónimos) y figuras inexistentes en otros ordenamientos y que no tienen una equivalencia exacta en el sistema de llegada.

Por ello, su estudio, en general, es pertinente en el ámbito de la traductología por las implicaciones que su traducción tiene. En este caso, además, se suma la particularidad del uso de una lengua no oficial de un país para la redacción de documentos que emanan de su administración, lo que no deja de ser un fenómeno digno de consideración desde el punto de vista (socio)lingüístico, traductológico y cultural. No hablamos de la confrontación de dos lenguas y dos culturas propia de cualquier proceso traductor. La diferencia, aquí, radica en el uso del francés para redactar determinados documentos cuya presentación (y traducción) es obligatoria para los ciudadanos marroquíes que deseen iniciar los trámites de residencia, reagrupación familiar, estudio, trabajo, etc. en España. No olvidemos que esta lengua no está reconocida como lengua oficial en Marruecos, lo que nos sitúa ante un escenario particular que algunos incluso tachan de ilegal.

\subsubsection{Consecuencias para la traducción}

$\mathrm{Al}$ margen de las cuestiones de índole política, cultural y social que puede acarrear el empleo de una lengua extranjera ${ }^{15}$ en la expedición de documentos emanantes de la Administración Pública de un Estado, existen cuestiones de corte lingüístico y traductológico relacionadas con este hecho que nos parece conveniente evocar.

\footnotetext{
${ }^{15}$ El uso del francés en textos procedentes de Marruecos podría interpretarse como el empleo de dicha lengua como lengua pivote entre el árabe y el español si consideremos que esta es la que opera entre la lengua origen y la lengua meta cuando, por alguna razón, no es posible (o no se desea) realizar esta operación directamente de la lengua A a la lengua B. Se trata, pues, de la presencia de un tercer elemento en una actividad (la traducción) inicialmente concebida para dos. Es una práctica frecuente en organismos internacionales, aunque el contexto no es el mismo, ya que, en algunos de ellos, como es el caso de la UE, existe un derecho comunitario que remite a conceptos comunes. Es decir, esta práctica se justifica por el contenido de los documentos que se traducen, que suele ser compartido desde un punto de vista referencial, ya que nacen en el seno de la institución, regida por un derecho y una normativa propias comunes a todos los estados miembros, lo que, en cierto modo, disminuye el desequilibrio cultural.
} 
En primer lugar, no podemos afirmar si los documentos objeto de traducción han sido traducidos del árabe al francés o si, por el contrario, se han corredactado en ambas lenguas en Marruecos. Desconocemos también (a no ser que dominemos ambas lenguas) hasta qué punto una versión es fiel a la otra y si esas traducciones/corredacciones son «oficiales» y constan como modelos en cada uno de los registros o no, aunque entendemos que lo normal (o, cuando menos, lo deseable) es que así sea. Sin embargo, lo que sí podemos constatar es la presencia de errores sintácticos, gramaticales y ortográficos detectados en la versión en francés de numerosos documentos registrales ${ }^{16}$, lo que nos lleva a preguntarnos por el proceso de redacción, corredacción y/o traducción de estos documentos.

En segundo lugar, los traductores jurados de francés no están obligados a conocer la lengua árabe; de hecho, la gran mayoría no tiene conocimiento lingüístico alguno sobre dicha lengua. Sin embargo, se ven en cierto modo beneficiados por el uso de la lengua francesa en los documentos registrales marroquíes, ya que la demanda de traducción de estos documentos es elevada. A pesar de ello, insistimos en el hecho de que, por un lado, se parte del empleo de una lengua no oficial en el país emisor (aunque dicho país admite esa práctica) y, por otro, la tentación de apartarse o, mejor dicho, de no acercarse a la cultura origen (la marroquí, en este caso) y de intentar asemejarla a otra más cercana o conocida (como la francesa, por ejemplo) podría provocar errores de traducción importantes.

\subsection{La traducción de textos registrales procedentes de Marruecos}

El presente trabajo pone el foco en los documentos registrales procedentes de Marruecos. Y ello por dos motivos fundamentales. El primero, porque, tal y como acabamos de exponer, la traducción de los documentos referidos es una constante para el traductor y, el segundo, porque presentan la particularidad de estar, en muchas ocasiones, redactados únicamente en francés o en versión bilingüe (francés y árabe), lo que, desde un punto de vista traductológico, es un fenómeno que no debe pasar desapercibido y cuyo análisis y consideración se nos antoja necesario por trascender lo meramente lingüístico. Para apoyar nuestras afirmaciones nos basamos en dos fuentes de información. La primera de ellas son los datos resultantes de la encuesta especialmente elaborada para este estudio, de la que a continuación se darán más detalles, y la segunda procede de la información de los organismos que establecen tanto los trámites de obligado cumplimiento para los marroquíes que, de forma más o menos estable y permanente, quieren permanecer en Espańa, como los documentos necesarios para ello, por

${ }^{16}$ Cabe señalar que estos errores suelen aparecer en aquella parte de los documentos que no varía, es decir, la que forma parte del formulario. Ello da a entender que, si se trata de un modelo establecido, los errores aparecerán siempre, lo que nos parece especialmente grave al tratarse de documentos expedidos por la Administración Pública. 
un lado, y de las entidades dedicadas a la tramitación de dicha documentación, por otro.

La justificación del volumen de trabajo que supone la traducción al español de documentos registrales procedentes de Marruecos se encuentra en las relaciones, a todos los niveles, que actualmente existen entre España y Marruecos y que determinan el flujo de personas entre ambos países por diversos motivos. Esta información queda avalada con las estadísticas sobre inmigración ofrecidas por el Instituto Nacional de Estadística español (INE, 2020) ${ }^{17}$. En consonancia con la encuesta realizada, se han extraído los datos relativos a España, Andalucía y la provincia de Málaga más actuales, con fecha de 1 de enero de 2020.

\begin{tabular}{|c|c|c|}
\cline { 2 - 3 } \multicolumn{1}{c|}{} & $\begin{array}{c}\text { Total de extranjeros } \\
\text { censados }\end{array}$ & $\begin{array}{c}\text { Total de marroquíes } \\
\text { censados }\end{array}$ \\
\hline Espańa & 5432198 & 812412 \\
\hline Andalucía & 700215 & 145076 \\
\hline Málaga & 269792 & 32781 \\
\hline
\end{tabular}

Tabla 1. Total de extranjeros y marroquíes censados en España,

Andalucía y Málaga según datos del INE a 1 de enero de 2020

Según los datos de la tabla 1, de todos los extranjeros censados en Andalucía, el $22,21 \%$ son marroquíes ${ }^{18}$. La marroquí es la primera nacionalidad en cuanto a número de extranjeros censados en España y Andalucía, y la segunda en Málaga.

Cada uno de los propósitos que se pretendan alcanzar (obtención de la nacionalidad, búsqueda de un trabajo, realización de estudios, reagrupación familiar, etc.) exigirá la realización de unos determinados trámites, marcados por la presentación de la documentación necesaria, que, en muchos casos, deberá obligatoriamente presentarse traducida (por un traductor jurado la mayoría de las veces) al español.

A partir de la información obtenida por las embajadas, el Ministerio de Asuntos Exteriores, Unión Europea y Cooperación (MAE, sd) y algunas agencias encargadas de realizar trámites de extranjería, y tomando como base los propósitos mencionados en el apartado anterior, los documentos cuya traducción se solicita con más frecuencia son los siguientes ${ }^{19}$ : certificado de nacimiento, certificado de matrimonio, certificado de parentesco, certificado de concordancia de nombre, certificado de soltería, certificado

${ }^{17}$ Cabe indicar que estos datos estadísticos, extraídos del sitio web del Instituto Nacional de Estadística (INE) son datos provisionales a 1 de enero de 2020 según la propia institución.

18 Para más información, consúltese el sitio web del INE (2020), que proporciona más datos y gráficos al respecto.

${ }^{19}$ Nótese que la mayoría de los documentos mencionados son registrales. Cabe aclarar que la lista se refiere a la documentación de carácter más personal, es decir, la que atañe directamente al estado de cada persona. Existen relaciones (de tipo comercial, por ejemplo) que dan lugar a la solicitud, expedición y consiguiente traducción de otros tipos de documentos registrales, como pueden ser los que emanan del Registro Mercantil (nota simple informativa, certificación negativa de denominación, etc.) o del Registro de la Propiedad. 
de divorcio o documentación que acredite la disolución de anteriores matrimonios, documento que certifique la patria potestad, certificado de antecedentes penales, certificado médico, título o diploma que acredite el acceso a los estudios o que indique los estudios seguidos, certificados bancarios, libro de familia, carta de admisión en el centro docente, copia de la autorización de residencia temporal por trabajo por cuenta ajena notificada al empleador y copia del contrato de trabajo.

\subsubsection{Resultados de la encuesta e interpretación de los datos obtenidos}

La encuesta aludida en el párrafo anterior se elaboró ad hoc para la ocasión y se envió a todos los traductores jurados de francés y de árabe de Andalucía incluidos en la lista del Ministerio de Asuntos Exteriores, Unión Europea y Cooperación de España (MAE, 2021): un total de $254^{20}$. A la encuesta, realizada durante los meses de noviembre y diciembre de 2019, se obtuvieron 46 respuestas (casi un $20 \%$ del total contactado), de los cuales 39 eran traductores jurados de francés y 7 de árabe ${ }^{21}$. El objetivo de nuestra encuesta era, fundamentalmente, obtener datos acerca del volumen y las condiciones de trabajo relacionados con la traducción de textos registrales, por una parte (preguntas 2, 3 y 4), y de la opinión de los traductores sobre el uso del francés en los textos registrales procedentes de Marruecos, por otra (preguntas 5 y 6 ).

Presentamos a continuación los resultados de las respuestas a algunas de las preguntas formuladas.

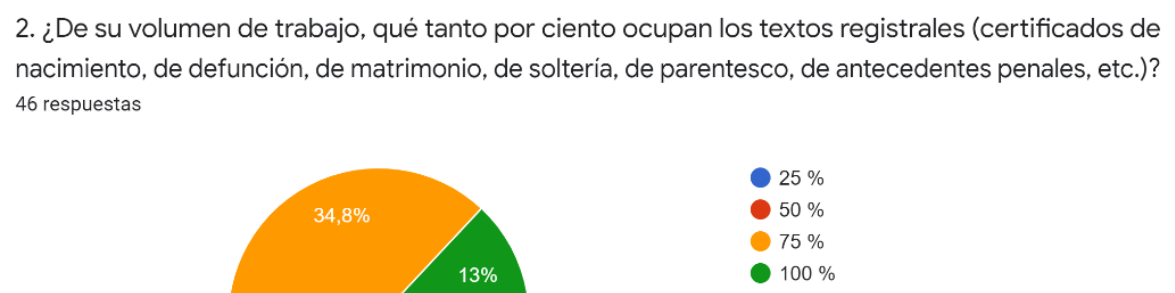

Gráfico 3. Resultados de la pregunta 2 de la encuesta realizada

\footnotetext{
${ }^{20}$ De esos 254, 28 no pudieron ser contactados debido a que los mensajes no llegaron a su destino (muchos traductores jurados no ejercen la profesión o no actualizan sus datos), lo que arroja un total de 226 traductores consultados (18 de árabe y el resto, 208, de francés).

${ }^{21}$ Aunque estas cifras puedan parecer poco representativas, debemos tener en cuenta que muchos de los traductores jurados que aparecen en las listas del Ministerio de Asuntos Exteriores, Unión Europea y Cooperación (MAE, 2021) no ejercen la profesión. Aun así, en el caso de los traductores de árabe obtuvimos respuesta del 39\% del total.
} 
3. ¿Suele recibir estos documentos en versión bilingüe (árabe y francés)?

46 respuestas

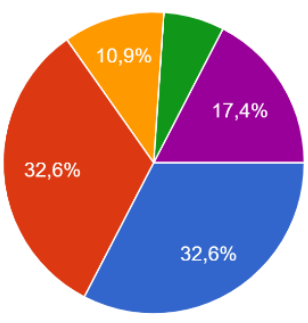

Sí, en un $25 \%$

Si, en un $50 \%$

Sí, en un $75 \%$

Si, en un $100 \%$

No

Gráfico 4. Resultados de la pregunta 3 de la encuesta realizada

4. ¿Ha debido usted traducir documentos que ya habian sido traducidos al español por un Traductor/a Jurado/a de Marruecos y que, por algún motivo, la traducción habia sido rechazada en España?

46 respuestas

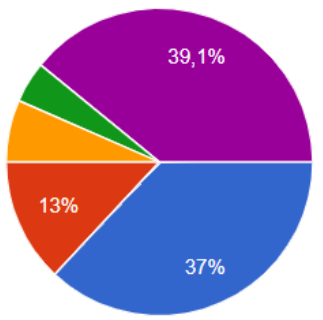

Si, en un $25 \%$

Sí, en un $50 \%$

Si, en un $75 \%$

Si, en un $100 \%$

No

Gráfico 5. Resultados de la pregunta 4 de la encuesta realizada

5. ¿Cree usted que en España se prefiere que los documentos procedentes de Marruecos vengan redactados en francés en lugar de en árabe para su traducción al español?

46 respuestas

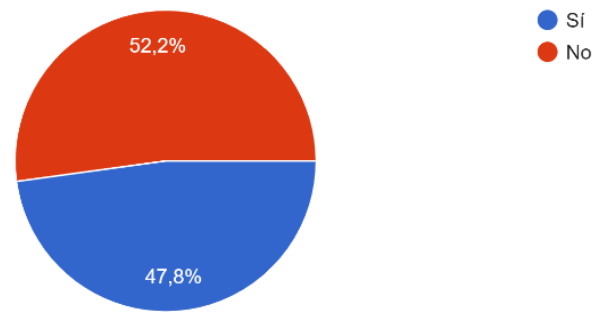

Gráfico 6. Resultados de la pregunta 5 de la encuesta realizada

De los datos obtenidos se infieren dos características relacionadas con aspectos lingüístico-traductológicos: primero, que los textos registrales representan un volumen de trabajo considerable de la carga total del traductor jurado, tal y como habíamos afirmado anteriormente, $y$, segundo, que la presencia del francés en dichos documentos es una realidad probada. 
Las preguntas 4 y 5 arrojan datos interesantes acerca de los problemas que surgen por la presentación de traducciones realizadas en Marruecos por traductores marroquíes y la actitud de la administración española, en general, ante la recepción de documentos procedentes de Marruecos en función de la lengua de redacción (francés o árabe).

En relación con este último aspecto, en la sexta y última pregunta de la encuesta («Si ha respondido afirmativamente a la pregunta 5, diga por qué cree que en España se prefiere que los documentos procedentes de Marruecos vengan redactados en francés en lugar de en árabe para su traducción al español») se obtuvieron respuestas diversas. La mayoría de ellas gira en torno a los siguientes factores:

- La traducción desde el árabe es más cara.

- Es más fácil encontrar un traductor jurado de francés que de árabe ${ }^{22}$.

- El árabe es una lengua que resulta más extraña para el público español.

Algunos de los encuestados, sin embargo, apuntaron razones de otra índole que se refieren a aspectos más delicados o subjetivos. Así, destacamos las respuestas proporcionadas por cuatro de los traductores preguntados. El traductor 1 afirmó que «en cuanto a la administración, se sienten más seguros al tener más control sobre el texto, ya que en un momento dado pueden cotejar la traducción y el original y ver que se corresponden, por ser el francés un idioma mucho más cercano al espańol». Para el traductor 2, «al Estado espańol poco le importa si la lengua de origen es una u otra, ya que obligan a su traducción al español». El traductor 3 subraya la necesidad de «informar a los marroquíes de que las traducciones de Marruecos no sirven en España». Por último, el traductor 4 afirma que «en general, existe cierta desconfianza (existe un mercado paralelo en el que los traductores no poseen títulos oficiales)».

La respuesta del traductor 2 (apuntada también por otros encuestados) parece dejar de manifiesto el desconocimiento de la administración, en general, sobre la traducción, su dificultad y sus características, ya que pone el foco en el resultado (el texto traducido) sin importarle, aparentemente, el proceso, hecho que no favorece en absoluto al traductor. El aspecto evocado por el traductor 3 tiene diferentes lecturas. Por un lado, está relacionado con la deontología del traductor cuando este es conocedor de una realidad que le puede beneficiar pero que perjudica al cliente. Por otro, muestra, en parte, la desconfianza ante la realización de traducciones hechas por traductores que no posean el título otorgado por el Ministerio de Asuntos Exteriores espańol. Esta desconfianza podría estar motivada por el hecho de que el acceso al nombramiento de traductor jurado difiere mucho de un país a otro, hecho al que a continuación nos referiremos. En cuanto a la existencia de un mercado paralelo mencionada por el

${ }^{22}$ Esto lo corrobora la diferencia entre el número de traductores jurados de árabe con respecto al número de traductores jurados de francés incluidos en la lista actualizada de traductores/as - intérpretes jurados/as nombrados por el ministerio de Asuntos Exteriores y de Cooperación (MAE, 2021). 
traductor 4, debemos decir que, en efecto, y sin que ello implique que sea la práctica habitual, sí se han dado casos controvertidos en España (García, 2010).

En la práctica, lo cierto es que muchos de los documentos (registrales en este caso) procedentes de Marruecos están redactados en francés, o en francés y árabe, y terminan siendo traducidos en multitud de ocasiones por traductores jurados de francés, que en muy escasas ocasiones tienen conocimientos de la lengua árabe (y, por ende, de su cultura jurídico-administrativa).

Es precisamente este hecho el que debería llevar a un análisis más pormenorizado ya que, de algún modo, se está «traduciendo Marruecos» a través de una lengua que no es la propia; no es, ni siquiera, una lengua oficialmente reconocida en dicho país. Si traducir directamente de una lengua a otra (y, por lo tanto, de una cultura a otra) es una tarea complicada llena de barreras lingüísticas y culturales, hacerlo a través de una tercera lengua (que, necesariamente, remite o puede remitir a otra(s) cultura(s)) puede, en cierto modo, "contaminar» el proceso.

A pesar de las reformas constantes al respecto, el nombramiento de traductor jurado sigue siendo objeto de crítica y crispación entre los traductores en muchos Estados, entre ellos, España. Las diferencias existentes entre los países con respecto al acceso a dicho nombramiento crean, además, desequilibrios importantes y esa falta de uniformidad o de criterios compartidos ha creado un clima de desconfianza que ha llevado a algunos Estados a admitir únicamente aquellas traducciones realizadas por profesionales cuyo título les han conferido ellos mismos, desechando así cualquier nombramiento extranjero.

En Marruecos, el texto que regula la forma de acceder al nombramiento de traductor jurado es la Loi $n^{\circ}$ 50-00 relative aux traducteurs agréés près les juridictions, promulgada por el Dahir n ${ }^{\circ}$ 1-01-127 du 29 rabii I 1422 (22 juin 2001) (Royaume du Maroc, 2001a). Además de establecer la obligatoriedad de cumplir con determinados requisitos $^{23}$, esta ley exige la superación del examen de traductor jurado (concours des traducteurs agréés), así como la del examen final del periodo de prácticas (examen de fin de stage). Con respecto al primero, corresponde al ministère de la Justice y/o a la AJAT (Asociación de Traductores Jurados de Marruecos) convocarlo, pero la falta de periodicidad y la poca adecuación a la realidad del ejercicio de la profesión han provocado numerosas críticas e incluso la petición de abolir la ley en cuestión (Libération, 2012). En lo relativo al periodo de prácticas, este debe durar un año y se realiza en el despacho

\footnotetext{
${ }^{23}$ Tener la nacionalidad marroquí (o la de otro país con el que Marruecos tenga un acuerdo que autorice a estas personas a ejercer la profesión de traductor en Marruecos), tener un título de traductor expedido por un establecimiento universitario de Marruecos o un título equivalente reconocido, no haber sido condenado por un delito grave o menos grave (salvo si ya está rehabilitado) ni haber estado condenado por ninguna infracción disciplinaria por hechos contrarios al honor, la moral o probidad; disfrutar de sus derechos civiles, haber cumplido con las obligaciones que impone la ley marroquí en relación con el servicio militar; etc.
} 
de un traductor jurado que ya ostente el título. El problema es que, en muchos casos, la obtención de ese título por parte de los «tutores» es anterior a la normativa actual (lo que provoca que algunos tilden la situación de injusta o desigual). Los candidatos, además, alegan un alto porcentaje de personas que no superan el examen, lo que achacan a un interés por parte de los traductores que ya ejercen por frenar, de algún modo, el acceso a la profesión de nuevos traductores jurados.

La lista oficial de traductores jurados de Marruecos (AJAT, sd) incluye 269 traductores, divididos en 10 combinaciones lingüísticas, siendo las combinaciones árabefrancés (con 94 traductores) y árabe-español (con 69) los grupos más numerosos. En cuanto a la formación de dichos traductores, existe una aparente disparidad en lo que a los títulos de acceso se refiere. Así, son numerosas y variadas las titulaciones a partir de las cuales los traductores optan al nombramiento ${ }^{24}$ (derecho, traducción, lengua y literatura, biología), aunque destacan aquellos traductores que están en posesión del título de la École supérieure Roi Fahd de traduction ${ }^{25}$.

Todo ello podría, en parte, justificar el recelo del Estado español a admitir traducciones de documentos marroquíes que no están realizadas por un traductor jurado incluido en la lista del Ministerio de Asuntos Exteriores, a pesar de que en España también existe cierta disparidad de criterios.

\section{Consideraciones finales}

La realidad lingüística y profesional descrita en el presente trabajo debería hacernos reflexionar en profundidad acerca de las implicaciones que el uso del francés como lengua pivote entre el árabe y el español tiene a todos los niveles y con respecto a todas las partes implicadas en el proceso.

Los resultados obtenidos a partir de nuestro estudio nos llevan a realizar las siguientes observaciones. En primer lugar, y a pesar de la normativa, el francés se sigue perfilando como lengua influyente y, tal y como afirmaba Moustaoui Srhir (2007: 193) para el caso de Marruecos, se ha convertido en "[...] patrimonio cultural que se ha impuesto entre la población».

En segundo lugar, creemos que el uso del francés en los textos jurídico-administrativos marroquíes presenta luces y sombras. Por un lado, parece que España es más receptiva a la presentación de documentos redactados en francés (frente al árabe). Además, los traductores de francés pueden ver aumentado su volumen de trabajo por este hecho. Sin embargo, y más allá de la legitimidad de este uso, el traductor de francés se ve confrontado a una problemática que no es en absoluto banal, ya que, en muchas

\footnotetext{
${ }^{24}$ A este respecto, cabe señalar que en España no se exige una determinada titulación, sino simplemente estar en posesión de un título universitario equivalente a los actuales grados. En Marruecos no se establece un mínimo de años de estudio.

25 Para acceder a esta formación es necesario tener al menos una licence o unos estudios univer-sitarios acreditados de tres años como mínimo.
} 
ocasiones, no conoce la lengua árabe y no está familiarizado con la cultura marroquí, lo que, en cierto modo, lo aleja de la realidad origen y puede llevar a cometer errores importantes en el proceso traductor. Digamos que, en cierto modo, el traductor debería partir de la fuente original, tanto textual como culturalmente.

En tercer lugar, llama la atención que, en un proceso en el que forzosamente están implicados, al menos, dos lenguas y dos sistemas, y habida cuenta de la importancia de la traducción jurada, no haya un mayor entendimiento entre países. En ese sentido, pensamos que habría alternativas para hacer este proceso menos problemático. Entre ellas proponemos el intercambio de las listas oficiales de los traductores jurados legítimamente habilitados en cada país, la posible «acreditación» de los traductores jurados de una lengua en aquellos países para los que podría traducir o la creación de una normativa común que regule este aspecto, al menos en ámbitos como la Unión Europea. Todo ello podría contribuir a disminuir ese mercado paralelo al que antes aludíamos.

En cuarto lugar, y en consonancia con el párrafo anterior, las administraciones deberían estar más sensibilizadas ante una actividad que les afecta directamente como es la traducción, ya que esta se perfila como la pieza clave en los procesos migratorios y de regularización de la situación jurídica y administrativa de las personas.

En quinto y último lugar, y desde una perspectiva pedagógica, resulta a nuestro entender indispensable que desde el aula de traducción jurídica (es decir, de cara a la formación de futuros traductores) se asuman estas realidades y que, en consecuencia, los contenidos se adapten al mercado profesional, a sus características y a sus dificultades.

\section{REFERENCIAS BIBLIOGRÁFICAS}

ABBOU, André (1984): «La langue française, un pont entre le Maghreb et l'Europe ?». Französisch Heute, 15 : 2, 219-223.

ACHOUCHE, Mohamed (1981): «La situation socio-linguistique en Algérie», in Olivier Dabène (ed.), Langue et migrations. Grenoble, Presses Universitaires de Grenoble, 3949.

AfELI, Kossi Antoine (1990): «Le français d'Afrique, pour quoi faire ?», in André Clas \& Benoît Ouoba (eds.), Visages du français: variétés lexicales de l'espace francophone. París, John Libbey Eurotext, 5-10.

AGUESSIM El GHAZOUANI, Abdellatif (2013): «Aproximación a la traducción jurídico-administrativa de documentos del registro civil marroquí: certificaciones de nacimientos». Tonos digital: Revista electrónica de estudios filológicos, 25. URL: http://www.um.es/tonosdigital/znum25/secciones/estudios-01-registro_civil_marroqui.htm. 
AgUiLera MarTíN, Juan Antonio (2003): «Política y planificación lingüísticas». Interlingüistica, 14, 91-96.

AKOUAOU, Ahmed (1984): «Pourquoi le français et quel français au Maroc ?». Le Français dans le monde, 189, 27-28.

AKOUAOU, Ahmed (1997): «Les variétés linguistiques au Maroc. Statuts, usages et fonctions», in Mohamed Taifi [ed.], Voisinage. Mélanges en hommage à la mémoire de Kaddour CADI. Fez, Publications de la Faculté des Lettres et des Sciences Humaines, 11, 6788.

AJAT $=$ ASSOCIATION DES TRADUCTEURS AGRÉES PRÈS LES JURIDICTIONS (sd): Liste de tous les traducteurs assermentés. URL: http://www.atajtraduction.asso.ma/resultatrecherche.php.

Barceló Martínez, Tanagua \& Iván Delgado Pugés (2014): «Certificados de nacimiento francófonos: aspectos de su superestructura y traducción al español. Análisis a partir de un corpus textual». Sendebar, 25, 39-58. URL: http://revistaseug.ugr.es/index.php/sendebar/article/view/1658/2608.

BARCELÓ MARTíNEZ, Tanagua \& Victoria GARCÍA ALARCÓN (2018): «Nuevas tendencias en traducción jurídico-jurada (francés-español) como consecuencia de las nuevas realidades sociales. Reflexiones a partir de la experiencia profesional», in Pino Valero, Analía Cuadrado \& Paola Carrión [eds.], Nuevas tendencias en traducción: Fraseología, Interpretación, TAVy sus didácticas. Berna, Peter Lang, 259-290.

BENÍTEZ FERNÁNDEZ, Montserrat (2006): «Approche sur la politique linguistique au Maroc depuis l'indépendance». Estudios de dialectología norteafricana y andalusi, 10, 109-120.

BENÍTEZ FERNÁNDEZ, Montserrat (2012): «Un repaso a la política lingüística del Norte de África desde la descolonización». Anaquel de estudios árabes, 23, 69-81.

BENZAKOUR, Fouzia (2010): «Le français au Maroc : enjeux et réalité». Le Français en Afrique, $25,33-41$.

BENZAKOUR, Fouzia (2012): «Le français au Maroc ; une variété occultée en quête de légitimité». Ponti/Ponts. Langues, littératures, civilisations des pays francophones, 12, 113-131.

BORJA ALBI, Anabel (2007): Estrategias, materiales y recursos para la traducción jurídica inglésespañol. Castellón, Universitat Jaume I, Servei de Comunicació i Publicacions.

BOURDEREAU, Frédéric (2006): «Politique linguistique, politique scolaire : la situation du Maroc». Le français aujourd'hui, 154: 3, 25-34.

BOUTEFFAH AGHBALOU, Bouchra (2017): La didáctica de las traducciones jurídica y jurada en la combinación lingüistica árabe-español: propuesta de un manual didáctico. Tesis doctoral dirigida por Nicolás Roser Nebot. Málaga, Universidad de Málaga. URL: https://riuma.uma.es/xmlui/bitstream/handle/10630/16235/TD_BOUTEFFAH_AGHBALOU_Bouchra.pdf?sequence=1\&isAllowed $=\mathrm{y}$.

BOUZIANE, Abdelmajid (2018): «L'enseignement de langues au Maroc : état des lieux et perspectives». Bassamat, 8, 27-52. URL: https://www.researchgate.net/publication/325533361_L'enseignement_des_langues_au_Maroc_etat_des_lieux_et_perspective s/citations. 
CASAS CABIDO, Francisco Javier (2000): «Las dificultades de la traducción jurada de documentos registrales procedentes de países francófonos», in Association suisse des traducteurs, terminologues et interpretes (eds.), Actes du colloque La traduction juridique : histoire, théorie(s) et pratique. Ginebra, Université de Genève, 503-519. URL: http://www.tradulex.com/Actes2000/casas.pdf.

Change.org (sd): Modification de la loi 50.00 des traducteurs assermentés au Maroc. URL: https://www.change.org/p/institution-du-m\%C3\%A9diateur-maroc-suppression-lala-loi-d\%C3\%A9cadente-et-discriminatoire-50-00-traducteurs-asserment\%C3\%A9s-au-maroc.

DE MigUel APARICIO, Elena (2000): «El texto jurídico-administrativo: análisis de una orden ministerial». Círculo de lingüistica aplicada a la comunicación, 4, s.p. URL: https://webs.ucm.es/info/circulo/no4/demiguel.htm.

DE POLI, Barbara (2005): «Francisation et arabisation au Maroc : l'identité linguistique entre enjeux symboliques et idéologiques». AION, 65: 1-4, 1-26.

El COURI, Mostapha (2013) : Histoire de la langue française au Maroc depuis 1912. Tetuán, Imprimerie Tétouan.

EL-MADKOURI MAATAOUI, Mohamed \& Beatriz SOTO ARANDA (2012): «Aspectos lingüísticos y extralingüísticos de la traducción jurídico-administrativa de documentos africanos en francés». Synergies Espagne, 5, 111-128. URL: https://gerflint.fr/Base/Espagne5/el_madkouri.pdf.

EL-MADKOURI MAATAOUI, Mohamed (2010): «Aspectos lingüísticos y extralingüísticos de la traducción jurídico-administrativa del árabe al español», in Saad Mohamed Saad \& María Katija Torres Calzada [coords.], Estudios de lingüistica y traductología árabe. Madrid, Instituto Egipcio de Estudios Islámicos, 229-263.

FADDI, Hicham (2015): Traducción para la inmigración. La multimodalidad en campañas de sensibilización dirigidas a inmigrantes marroquies. Tesis doctoral dirigida por Ovidi Carbonell i Cortés. Salamanca, Universidad de Salamanca.

FERIA GARCÍA, Manuel C. (1999): «La traducción jurada de actas matrimoniales marroquíes», in Manuel Feria García (ed.), Traducir para la justicia. Granada, Comares, 221-258.

FERNÁNDEZ VÍTORES, David \& Fatiha BENLABBAH (2014): La lengua española en Marruecos. Rabat, UM5 Rabat. URL: http://www.exteriores.gob.es/Embajadas/RABAT/es/Noticias/Documents/LENGESPMARR.pdf.

GARCÍA, Jesús (2010): «La policía destapa una red de falsos traductores de árabe en Barcelona». El Pais, 1 de abril de 2010. URL: https://elpais.com/diario/2010/04/01/espana/1270072807_850215.html

INE = INSTITUTO NACIONAL DE ESTADÍSTICA (2020): Demografía y población. Cifras de población y censos demográficos. URL: https://www.ine.es/dyngs/INEbase/es/categoria.htm?c=Estadistica_P\&cid=1254734710984.

KADIRI HASSANI, Hasnaa (2015): La traduction au Maroc sous le protectorat français (19121956). Etude de trois auto-traducteurs de l'IHEM. Tesis doctoral dirigida por Georges 
Bastin. Montreal, Université de Montréal. URL: https:/pdfs.semanticscholar.org/1096/2ce29e2837cbcacac431e9175323d35f720d.pdf.

KARIM, Hasna (2010): La traducción de los anisomorfismos culturales en el ámbito jurídico. Aplicaciones al derecho marroqui en comparación con el derecho francés y español. Tesis doctoral dirigida por Emilio Ortega Arjonilla. Málaga, Universidad de Málaga. URL: https://riuma.uma.es/xmlui/bitstream/handle/10630/4676/TDR_KA-

RIM_HASNA.pdf?sequence $=6 \&$ isAllowed $=\mathrm{y}$.

LE SITE INFO (2018): La langue française jugée illégitime dans les administrations au Maroc. URL: https://www.lesiteinfo.com/maroc/la-langue-francaise-jugee-illegitime-dansles-administrations-au-maroc/.

LIBÉRATION (2012): Examen d'assermentation près les juridictions: Bisbille dans la corporation des traducteurs. URL: https://www.libe.ma/Examen-d-assermentation-pres-les-juridictions-Bisbille-dans-la-corporation-des-traducteurs_a33431.html.

MANZANO, Francis (1996): «Sur les mécanismes du paysage sociolinguistique et identitaire d'Afrique du nord». Langage et société, 75, 5-43.

MESSAOUIDI, Leila (2010): «La langue française au Maroc, fonction élitaire ou utilitaire ?», in Philippe Blanchet \& Pierre Martinez (eds.), Pratiques innovantes du plurilinguisme, émergence et prise en compte en situations francophones. París, Éditions des archives contemporaines, 51-63.

MAE $=$ MINISTERIO DE ASUNTOS EXTERIORES, UNIÓN EUROPEA Y COOPERACIÓN (sd): Información para extranjeros. URL: http://www.exteriores.gob.es/Portal/es/ServiciosAlCiudadano/InformacionParaExtranjeros/Paginas/Trabajar.aspx.

MINISTERIO DE ASUNTOS EXTERIORES, UNIÓN EUROPEA Y COOPERACIÓN (2021): Lista actualizada de traductores/as - intérpretes jurados/as nombrados por el Ministerio de Asuntos Exteriores y de Cooperación. URL: http://www.exteriores.gob.es/Portal/es/ServiciosAlCiudadano/Documents/Lista\%20actualizada\%20a\%2026\%20marzo\%202021.pdf.

MOUSTAOUI SRHIR, Adil (2007): Lenguas, identidades y discursos en Marruecos: la pugna por la legitimidad. Tesis doctoral bajo la dirección de Carles Castellanos i Llorens. Barcelona, Universitat Autònoma de Barcelona.

ORGANISATION INTERNATIONALE DE LA FRANCOPHONIE (2018): Langue française et diversité linguistique. URL: http://observatoire.francophonie.org/qui-parle-francais-dansle-monde.

PARAINMIGRANTES.INFO (2015): ¿Qué es un registro público? URL: https://www.parainmigrantes.info/que-es-un-registro-publico-843.

PÉREZ DE GRACIA SÁNCHEZ, Laura (2018): La traducción al español de documentos registrales marroquies redactados en francés: caracterización, descripción y análisis a partir de un corpus textual. Trabajo Fin de Grado. Málaga, Universidad de Málaga.

QUITOUT, Michel (2001): «L'arabe, le français, l'amazighe au Maroc : un patrimoine culturel national». Cahiers du Rifal, 22, 60-65. URL: http://termisti.ulb.ac.be/archive/rifal/PDF/rifal22/rifal22_Quitout.pdf. 
ROYAUME DU MAROC (1999): Charte nationale d'éducation et de formation. URL: http://www.mcinet.gov.ma/sites/default/files/documentation\%20iscae\%20rabat\%202018.pdf.

ROYAUME DU MAROC (2000): Loi no 01-00 portant l'organisation de l'enseignement supérieur du 1er juin 2000. URL: http://www.umi.ac.ma/wp-content/uploads/2012/03/loi-n01-00-portant-organisation-de-lenseignement-sup\%C3\%A9rieur.pdf.

ROYAUME DU MAROC (2001a): Dahir no 1-01-127 portant la promulgation de la loi no 50-00 relative aux traducteurs agréés près les juridictions, du 22 juin 2001 (29 rabii I 1422)). URL: http://adala.justice.gov.ma/production/html/Fr/67740.htm.

ROYAUME DU MAROC (2001b): Dahir no 1-01-299 portant création de l'Institut royal de la culture amazigue (IRCAM), du 17 de octobre 2001 (29 rajab 1422). URL: http://www.axl.cefan.ulaval.ca/afrique/maroc-dahir2001.htm.

ROYAUME DU MAROC (2011). Constitution du Maroc en français. URL: http://www.sgg.gov.$\mathrm{ma} /$ Portals/0/constitution/constitution_2011_Fr.pdf.

ROYAUME DU MAROC (2019): La réforme administrative au Maroc. URL: https://uclgafricaalga.org/wp-content/uploads/2019/05/unpan002395.pdf.

RUIZ CORTÉS, Elena (2019): «Comparación de la traducción de recursos digitales en un procedimiento de extranjería en Espańa y en el Reino Unido: un estudio de caso». Revista de Llengua i Dret - Journal of Language and Law, 71, 223-237. DOI: https://doi.org/10.2436/rld.i71.2019.3245. 